\title{
THE FORMATION KIMBERLITE DIAMONDS THROUGH CHEMICAL SYNTHESIS IN OPEN CATALYTIC SYSTEM.
}

Kulakova, I.I.; Rudenko, A.P. and Skvortsova, V.L.

Chemical Department, Moscow State University, 119899, USSR.

The existence of natural diamond of various genetic types and successful synthesis of diamond show the possibility of various mechanisms of diamond formation. All possible processes of diamond formation may be divided into two groups: crystallization processes during polymorphic transformation of graphite to diamond under high P,T-conditions and processes of chemical synthesis of diamond from low carbon-containing molecules under relatively mild $\mathrm{P}, \mathrm{T}$-conditions.

We consider the problem of kimberlite diamonds formation in the context of general laws of chemistry, thermodynamics and catalysis taking into account the theory of open catalytic systems and geochemical features of diamond occurrence in kimberlites.

Hereby we suggest a model in which the formation of kimberlite diamonds result from catalytic polycondensation of simple carbon-containing substances in non-stationary open catalytic systems, such as in the following reactions:

$$
\begin{aligned}
& 2 \mathrm{CO} \rightleftarrows \mathrm{C}_{\mathrm{d} \text { iamond }}+\mathrm{CO}_{2} \\
& \mathrm{CO}+\mathrm{H}_{2} \rightleftarrows \mathrm{C}_{\mathrm{d} \text { iamond }}+\mathrm{H}_{2} \mathrm{O} \\
& \mathrm{CO}_{2}+\mathrm{CH}_{4} \rightleftarrows 2 \mathrm{C}_{\mathrm{diamond}}+2 \mathrm{H}_{2} \mathrm{O} \\
& \mathrm{CO}_{2}+4 \mathrm{FeO} \rightleftarrows \mathrm{C}_{\text {diamond }}+2 \mathrm{~F}_{2} \mathrm{O}_{3}
\end{aligned}
$$

Each of these processes has an aromatic polycondensation analogue that eventually produces under the same $\mathrm{P}, \mathrm{T}$-conditions: graphite, bitumens and others products. Thermodynamic conditions and catalysts for diamond and graphite formation are similar, but the oxidizing medium potential and the rates of these processes are different.

According to the model one can distinguish two stages the macroscopic diamond crystals formation. The first one formation of diamond ("germs") can proceed either in a static system (up to the equilibrium) or in the open system in non-equilibrium conditions. And diamond "germ" forms as a result of polycondensation of carbon-containing substances in the form of epitaxial films on planes of some minerals, containing catalytically active ions for diamond polycondensation ( $\mathrm{Cr}, \mathrm{Mn}, \mathrm{Fe}$ and others) and having crystallostructural correspondence with the diamond lattice. This is in good accordance with the "central" inclusi- 
ons in diamond crystals and with the regularities of their orientation.

The second stage (up to the formation of macroscopic crystals) is possible only in open non-equilibrium systems when the initial substances ( $\mathrm{CO}, \mathrm{CO}+\mathrm{H}_{2}$ and others) cause gradual growth of crystal and the movable products $\left(\mathrm{CO}_{2}, \mathrm{H}_{2} \mathrm{O}\right)$ are gone, the equilibrium of the above-mentioned reactions being shifted to the right.

The main components of such an open system are growing crystals and the surrounding mineral coat, containing ions-catalysts of diamond growth/oxidation ( $\mathrm{Cr}, \mathrm{Fe}, \mathrm{Mn}, \mathrm{Ni}$ and others). Increasing size of diamonds or their oxidation are determined by the medium redox potential and temperature.

The following facts support this mode1:

- decrease in the content of diamonds in kimberlite tubes with depth;

- difference in the content and morphology of diamond in different zones of the same tube;

- existence of tubes either continuous diamonds or lacking them within one and the same region;

- catalytic activity of kimberlites in the oxidation-growth processes of diamonds;

- present of mineral coats on the diamond crystals and their physico-chemical and mineralogical peculiarities;

- correspondence between diamond contents of kimberlites and their catalytic activities, chemical composition and contents bi tumens. 